\title{
Mitigation of Pattern Sensitivity in Full-Field Electronic Dispersion Compensation
}

\author{
J.Zhao, M.E.McCarthy, P.Gunning*, A.D.Ellis
}

\begin{abstract}
We investigate the pattern-dependent decoding failures in full-field electronic dispersion compensation (EDC) by off-line processing of experimental signals, and find that the performance of such an EDC receiver may be degraded by an isolated ' 1 ' bit surrounded by long strings of consecutive ' 0 's. By reducing the probability of occurrence of this kind of isolated ' 1 ' and using a novel adaptive threshold decoding method, we greatly improve the compensation performance to achieve $10 \mathrm{Gbit} / \mathrm{s}$ on-off keyed signal transmission over $496 \mathrm{~km}$ field-installed single-mode fibre without optical dispersion compensation.
\end{abstract}

Index Terms - Modulation, chromatic dispersion, electronic dispersion compensation, detection

\section{INTRODUCTION}

$\mathrm{E}$ LECTRONIC dispersion compensation (EDC) has attracted much interest recently due to its cost effectiveness and adaptive equalization capability [1-2]. Receiver-side EDC, which can adapt quickly to changes in link conditions, is of particular value for future transparent optical networks with many reconfigurable add- and drop-nodes. However, the performance of conventional EDC using direct detection (DD EDC) is limited due to loss of the signal phase information [1]. EDC based on coherent detection can access both the intensity and the phase of the optical field, allowing for full chromatic dispersion (CD) compensation [2]. However, it may require a complicated optical implementation in order to achieve polarization and phase tracking between the local oscillator and the signal.

We have recently proposed a novel EDC receiver in 10Gbit/s on-off keyed (OOK) systems, which can access the optical intensity and phase information simultaneously, using a single asymmetric Mach-Zehnder interferometer (AMZI) [34]. The recovered information allows for full-field $C D$ compensation using a dispersive microwave transmission line [3] or full-field maximum likelihood sequence estimation [4]. In the dispersive transmission line based system, this scheme was experimentally verified to successfully recover a $10 \mathrm{Gbit} / \mathrm{s}$ OOK signal after transmission over $248 \mathrm{~km}$ of field-installed

Manuscript received July 28, 2008. This work was supported by Science Foundation Ireland under grant number 06/IN/I969.

J.Zhao, M.E.McCarthy, A.D.Ellis are with Photonic Systems Group, Tyndall National Institute, University College Cork, Lee Maltings, Prospect Row, Cork, Ireland (e-mail: jian.zhao@tyndall.ie).

P.Gunning is with Futures Testbed, BT Design, Adastral Park, Martlesham Heath, Ipswich, UK (e-mail: paul.gunning@bt.com).

Copyright C 2008 IEEE single mode fibre (SMF) [3]. In this paper, we show that for transmission distances over $250 \mathrm{~km}$, the performance of fullfield EDC receiver is degraded by an isolated ' 1 ' bit surrounded by long strings of consecutive ' 0 's. By reducing the probability of occurrence of this kind of isolated ' 1 ' and using a novel adaptive threshold decoding (ATD) method, we substantially reduced the pattern-dependent decoding failures and achieved 10Gbit/s OOK signal transmission over $496 \mathrm{~km}$ field-installed SMF without optical dispersion compensation.
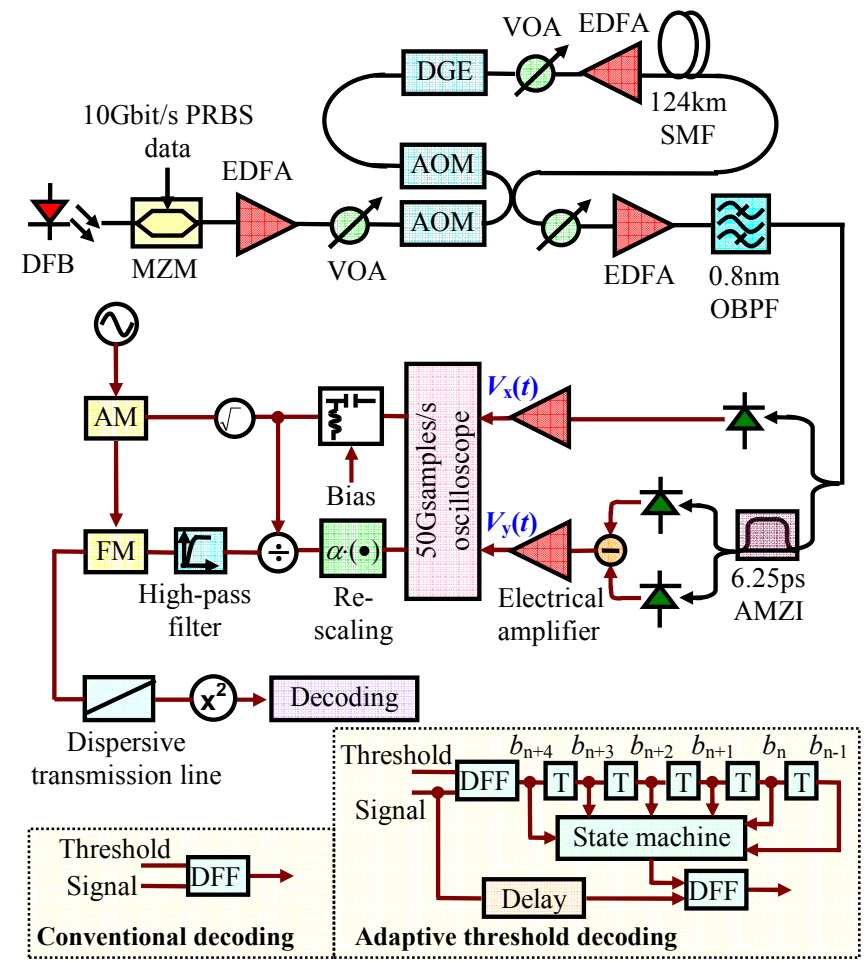

VOA: variable optical attenuator; EDFA: erbium doped fiber amplifier;

AOM: acousto-optical modulator; DGE: dynamic gain equalizer

Fig. 1. Experimental setup

\section{EXPERIMENTAL SETUP AND PRINCIPLE}

Fig. 1 shows the experimental setup. A $1557 \mathrm{~nm}$ signal from a distributed feedback laser (DFB) was intensity modulated using a Mach-Zehnder modulator (MZM) giving a $12 \mathrm{~dB}$ extinction ratio (ER) signal at $10 \mathrm{Gbit} / \mathrm{s}$ with an either $2^{7}-1$ or $2^{11}-1$ pseudo-random binary sequence (PRBS). The OOK signal that resulted was transmitted over a recirculating loop comprising $124 \mathrm{~km}$ of BT Ireland's field-installed SMF between Cork City and Clonakilty, County Cork, Ireland, with a signal launch power of around $0 \mathrm{dBm}$ per span. The SMF has 
$\mathrm{CD}$ of around $16 \mathrm{ps} / \mathrm{km} / \mathrm{nm}$ and a total loss of $25 \mathrm{~dB}$. At the receiver, the signal was pre-amplified and optically filtered by a $0.8 \mathrm{~nm}$ optical bandpass filter (OBPF). Ten percent of the signal power was directly detected by a $10 \mathrm{Gbit} / \mathrm{s}$ receiver and electrically amplified by a $12 \mathrm{GHz}$ electrical amplifier. The remaining signal was optically processed by an AMZI with a $6.25 \mathrm{ps}$ differential time delay and $\pi / 2$ differential phase shift, detected by a $40 \mathrm{GHz}$ balanced detector, and electrically amplified with a net amplification bandwidth of $15 \mathrm{GHz}$. Both detected signals were sampled by a real-time oscilloscope at 50Gsamples/s. Off-line processing with 4,000,000 samples was used for optical intensity and phase recovery using the method outlined in [3]. $V_{\mathrm{x}}(t)$ was re-biased to allow for the $\mathrm{AC}$ coupling of the receiver. $V_{\mathrm{y}}(t)$ was re-scaled to correct for different gains of the $V_{\mathrm{x}}(t)$ and $V_{\mathrm{y}}(t)$ signal paths. A Gaussianshaped high-pass filter with $1.2 \mathrm{GHz} 3 \mathrm{~dB}$ cut-off frequency was placed in the frequency estimation path to improve the quality of phase estimation and accordingly the compensation performance [3-4]. The performance improvement obtained using the high-pass filter is illustrated in Fig. 2(a) and (b), where the compensated signal with a $2^{11}-1$ sequence at a fibre length of $496 \mathrm{~km}$ and $26 \mathrm{~dB}$ OSNR shows significantly clearer eyes when using the high-pass filter. The compensated microwave signal was square-law detected and decoded by either conventional threshold decoding or the proposed ATD.
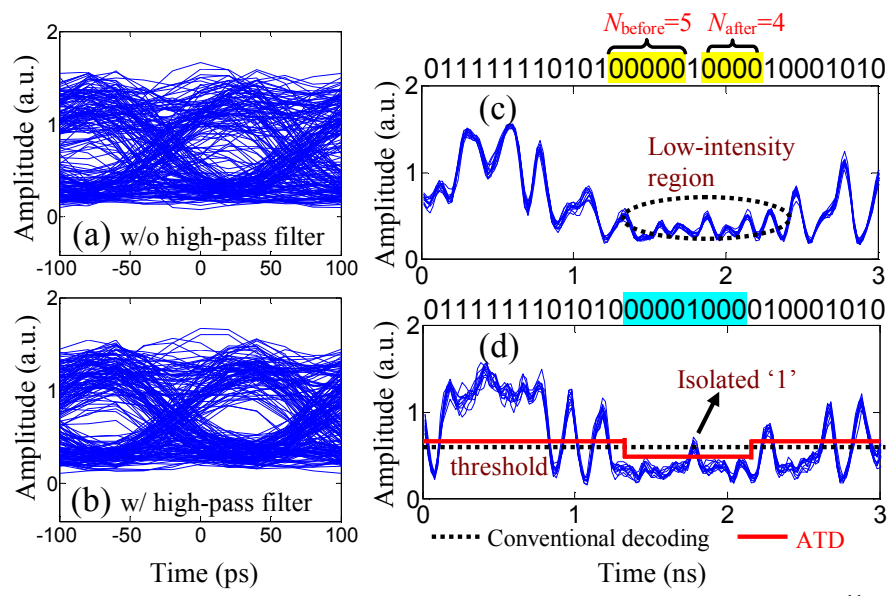

Fig. 2. (a) and (b): Eye diagrams of the compensated signal with $2^{11}-1$ sequence at $496 \mathrm{~km}$ and $26 \mathrm{~dB}$ OSNR (a) without and (b) with a $1.2 \mathrm{GHz}$ highpass filter; (c) and (d): a data sequence that contains an isolated ' 1 ' in a signal with $2^{11}-1$ sequence at $496 \mathrm{~km}$ (c) without and (d) with EDC.

Fig. 2 (c) and (d) illustrate the experimental intensity captures of a data sequence that contains an isolated ' 1 ' (c) without and (d) with EDC after 496km SMF transmission. $N_{\text {before }}$ and $N_{\text {after }}$ represent the number of consecutive ' 0 's before and after the isolated ' 1 ' respectively. From Fig. 2(c), it can be seen that a typical isolated ' 1 ' following $496 \mathrm{~km}$ transmission results in a low optical intensity in that region. Consequently, it is expected that the poor signal-to-noise ratio would increase the uncertainty in both the extracted intensity and the instantaneous frequency, which would hinder the reconstruction of such isolated ' 1 ' bit. As a result, the isolated ' 1 ' might result in erroneous decisions, even after EDC if conventional threshold detection is used (Fig. 2(d)).
This pattern sensitivity may be alleviated by using appropriate line coding in a practical system to reduce the number of such sequences at the expense of a certain amount of overhead. In order to avoid this overhead, a receiver-side mitigation technique may be used to adaptively use a lower threshold for sequences containing a single isolated ' 1 ' and a higher threshold elsewhere, as proposed here. This ATD can be viewed, to a certain extent, as a simple line decoder without the sacrifice of net data rate and the transmitter-side encoder module. As illustrated in Fig. 1, a D-flip flop (DFF) was used to perform an initial bit estimate, with the output data evaluated by a state machine. The state machine has two states representing two output values and is initially preset at state ' 1 ' with output of threshold ' 1 '. When a sequence containing a single isolated ' 1 ' occurs, the first DFF may erroneously detect it as a long string of consecutive ' 0 's due to low intensity of the isolated ' 1 '. The state machine determines if the initial estimate suggests a chain of consecutive ' 0 ' bits and under this condition changes its state to give threshold ' 2 ' in order to allow the detection of low-intensity isolated ' 1 ' bits. State ' 2 ' is held until the sequence of consecutive ' 0 's ends. Table 1 summarizes the principle.

TABLE 1: TRUth TABle OF ADAPTIVE Threshold DeCODING

\begin{tabular}{|l|l|l|l|}
\hline State & Input & Action & Output \\
\hline \multirow{3}{*}{ State ' 1 ' } & $b_{\mathrm{n}-\mathrm{m}} \ldots \& b_{\mathrm{n}} \& \ldots b_{\mathrm{n}+\mathrm{k}}=0$ & Change state & Threshold '2' \\
\cline { 2 - 4 } & Else & Hold state & Threshold '1' \\
\hline \multirow{2}{*}{ State '2' } & $b_{\mathrm{n}+\mathrm{m} \neq 0} \neq 0$ & Change state & Threshold '1' \\
\cline { 2 - 4 } & $b_{\mathrm{n}+\mathrm{m}}=0$ & Hold state & Threshold '2' \\
\hline
\end{tabular}

In the table, $b_{\mathrm{n}}$ represents the logical data from the first DFF. $m$ and $k$ determine the conditions which the decoder recognizes as a long string of consecutive ' 0 's and were set to be 1 and 4 respectively in this paper. The adaptive threshold obtained by the state machine is fed into the second DFF to compare with the properly delayed signal. An example illustrating the operation of ATD is shown in Fig. 2(d).

\section{RESULTS AND DISCUSSIONS}

In the off-line processing, the parameters, including bias added to $V_{\mathrm{x}}(t)$, scaling factor of $V_{\mathrm{y}}(t)$, compensation ratio and thresholds ' 1 ' and ' 2 ', were adjusted to achieve a local minimal rather than the global minimal bit error rate (BER) for each set of data, due to complexity of global optimization.

As a figure of merit, we define a normalized error probability $(N E P)$ of a pattern as:

$$
N E P_{\text {pattern }}=\frac{N E_{\text {pattern }}}{N E_{\text {sequence }}} \frac{N B_{\text {sequence }}}{N B_{\text {pattern }}}
$$

where $N E_{\text {pattern }}$ (or $N B_{\text {pattern }}$ ) represent the number of errors (or bits) of the particular pattern in the observed sequence, whilst $N E_{\text {sequence }}$ (or $N B_{\text {sequence) }}$ ) represent $N E$ (or $N B$ ) of the whole sequence. $N E P_{\text {pattern }}$ will be greater than one if a given pattern causes abnormally high errors. Fig. 3(a) shows the occurrence probability of a pattern with a given degree of ' 1 ' bit isolation $\left(\min \left(N_{\text {before }}, N_{\text {after }}\right)\right)$, whilst Fig. 3(b) shows $N E P$ following offline signal processing of the received signal. The dotted line in Fig. 3(b) is an arbitrary fitted curve for all symbols showing 
the general trends with respect to isolation. The figure shows that $N E P$ increases dramatically as the isolation increases to 4 after which it becomes steady, verifying the susceptibility of the EDC receiver to isolated ' 1 ' bits. Note that ' 1 ' bits with larger isolation $(\geq 4)$ occur less frequently in a signal with $2^{11}-1$ sequence, which should therefore lead to reduced BER compared to a signal with $2^{7}-1$ sequence. The occurrence probability of ' 1 ' bits with such large isolation in real data is $\sim 1 / 2^{9}$.
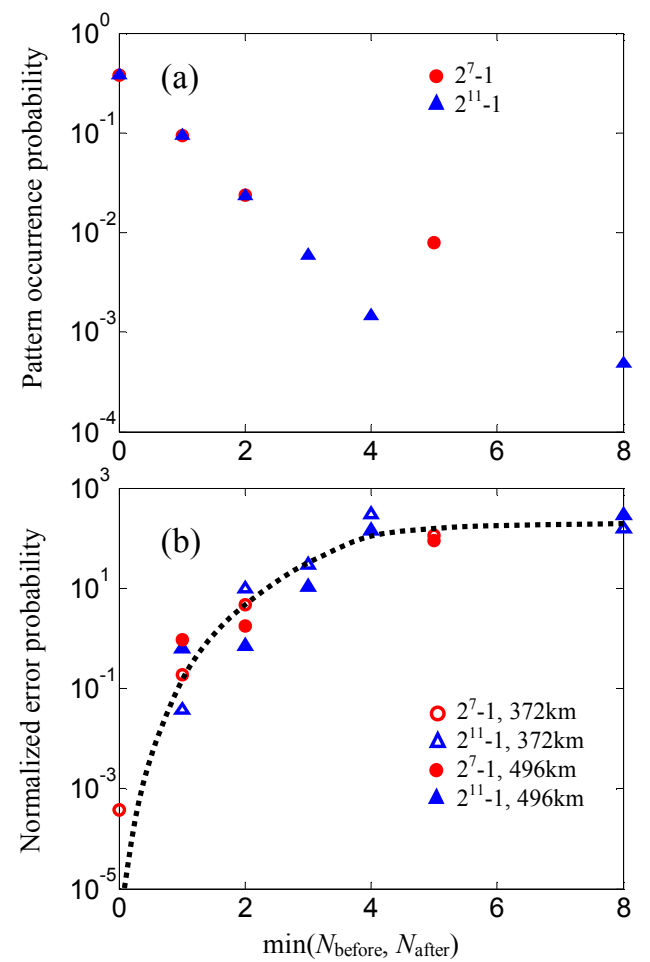

Fig. 3. (a) Pattern occurrence probability versus $\min \left(N_{\text {before }}, N_{\text {after }}\right)$ for $2^{7}-1$ (circles) and $2^{11}-1$ (triangles) sequences. (b) Normalized error probability as a function of $\min \left(N_{\text {before }}, N_{\text {after }}\right)$ for $2^{7}-1$ (circles) and $2^{11}-1$ (triangles) sequences after $372 \mathrm{~km}$ (open) and 496km (closed) SMF transmissions.

Fig. 4 depicts $\log _{10}(\mathrm{BER})$ versus OSNR $(0.1 \mathrm{~nm}$ resolution) after (a) $372 \mathrm{~km}$ and (b) $496 \mathrm{~km}$ of field-installed SMF transmission. From the figure, it is shown that by using $2^{7}-1$ sequence, $372 \mathrm{~km}$ SMF transmission was unachievable. Analysis of the error distribution (Fig. 3(b)) showed that most errors were caused by the isolated ' 1 ' with $\min \left(N_{\text {before }}\right.$, $\left.N_{\text {after }}\right)>2$. For a signal with $2^{11}-1$ sequence, the density of isolated ' 1 ' bit surrounded by long strings of ' 0 's was reduced, enabling a substantial performance improvement of full-field EDC with a $4 \times 10^{-4}$ BER at $26 \mathrm{~dB}$ OSNR after $372 \mathrm{~km} \mathrm{SMF}$ transmission. However, a significant number of errors were still induced by isolated ' 1 ' bits, which hindered further distance extension to $496 \mathrm{~km}$. By using a $2^{11}-1$ sequence and ATD described above, the errors from isolated '1's were further reduced. As a result, $496 \mathrm{~km}$ SMF transmission was achieved with a $3.3 \times 10^{-4}$ BER at $26 \mathrm{~dB}$ OSNR, representing an $8 \mathrm{~dB}$ penalty from the required back-to-back OSNR at this ER. Note that the experimental setup was not optimized, and performance improvement is predicted by using optimized optical and electrical component parameters [5].
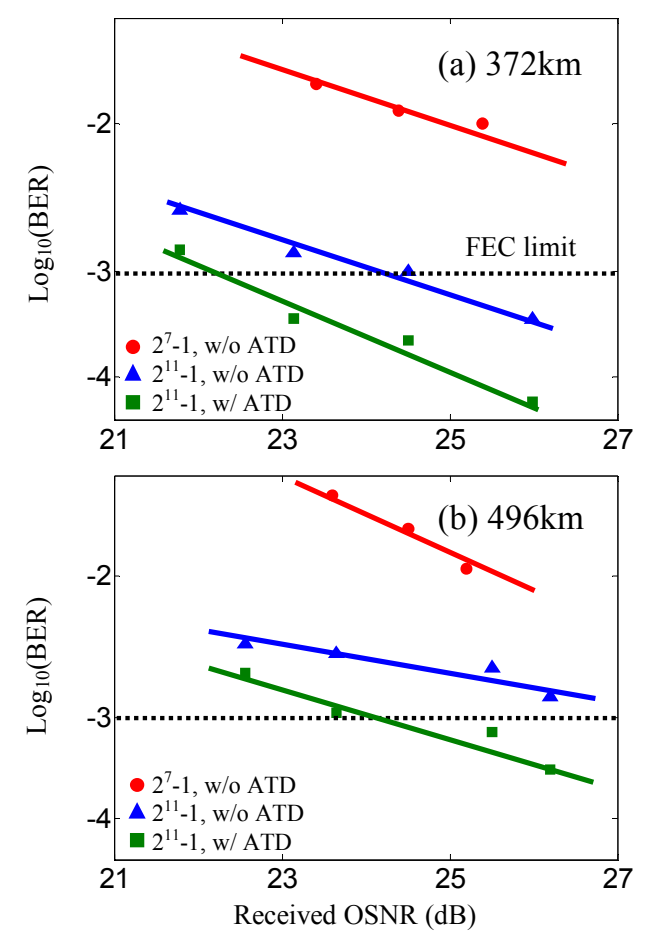

Fig. 4. $\log _{10}$ (BER) versus OSNR (0.1nm resolution) for (a) $372 \mathrm{~km}$ and (b) $496 \mathrm{~km}$ SMF transmission. Circles, triangles, and squares represent $2^{7}-1$ sequence with conventional decoding, $2^{11}-1$ sequence with conventional decoding, $2^{11}-1$ sequence with adaptive threshold decoding, respectively.

\section{CONCLUSION}

We have investigated the pattern-dependent decoding failures in full-field EDC, and found that the performance of such an EDC receiver was degraded by patterns of isolated ' 1 ' surrounded by long strings of consecutive ' 0 's. By reducing the probability of such patterns occurring and using a simple ATD, we have significantly improved the performance to achieve 10Gbit/s OOK signal transmission over $496 \mathrm{~km}$ of field-installed SMF without optical dispersion compensation.

\section{ACKNOWLEDGMENTS}

The authors gratefully acknowledge BT Ireland, particularly J.Coleman, M.Conroy, W.McAuliffe, R.O'Regan and M.O'Reilly, for providing access to the fibre link.

\section{REFERENCES}

[1]. H. Bulow, "Electronic dispersion compensation", in Proc. Optical Fiber Communication Conference (OFC), OMG5, 2007.

[2]. A. H. Gnauck, J. Sinsky, P. J. Winzer, and S. Chandrasekhar, "Line microwave-domain dispersion compensation of $10-\mathrm{Gb} / \mathrm{s}$ signals using heterodyne detection", in Proc. Optical Fiber Communication Conference (OFC), PDP31, 2005.

[3]. J. Zhao, M. E. McCarthy, P. Gunning, A. D. Ellis, "Dispersion tolerance enhancement in electronic dispersion compensation using full opticalfield reconstruction", in Proc. Optical Fiber Communication Conference (OFC), OWL3, 2008.

[4]. M. E. McCarthy, J. Zhao, P. Gunning, A. D. Ellis, "A novel fielddetection maximum-likelihood sequence estimation for chromaticdispersion compensation", European Conference on Optical Communication (ECOC), We.2.E.5, 2008.

[5]. J. Zhao, M. E. McCarthy, A. D. Ellis, "Electronic dispersion compensation using full optical field reconstruction in $10 \mathrm{Gbit} / \mathrm{s}$ OOK based systems", Opt. Express, vol. 16, pp.15353-15365, 2008. 